\title{
Cracking behavior of thin gold strip deposited on polycarbonate plate under Cyclic and stepwisely-increased tension
}

\author{
Naoya Tada ${ }^{1}$, Yafei Hu${ }^{2}$, Takeshi Uemori ${ }^{1}$ and Toshiya Nakata ${ }^{1}$ \\ ${ }^{1}$ Okayama University, Graduate School of Natural Science and Technology, Okayama, Japan \\ ${ }^{2}$ Qingdao University of Science and Technology, College of Mechanical and Electronical Engineering, Qingdao, Shandong, P.R. China
}

\begin{abstract}
Thin metal films deposited on polymers have been attracting much attention in flexible electronics. Various combinations of film and polymer substrate are expected to be developed. In this paper, deformation and fracture of thin metal film on polymer substrate was briefly analysed and the influencing factors were summarized. After that, focusing on the shape and thickness of thin metal film on polymer substrate, cracking behavior of small roundedrectangular thin gold films called "strips" with a thickness less than one micrometer was observed under cyclic and stepwisely-increased trapezoidal tensions. The strips were deposited on a polycarbonate plate specimen, and wide and narrow strips with different thickness were prepared for the tests. The cracking behaviour was successfully observed and the increasing rate of crack number with the load or strain was examined. Since most cracks were initiated from the small defects, the crack initiation behavior was correlated with the distribution of defects.
\end{abstract}

\section{Introduction}

Thin metal films on polymer substrates are commonly used in flexible electronics and expected to be developed not only in the electronics but also in biological and medical fields $[1,2]$. The mechanical performance of a thin metal film on polymer substrate is mainly determined by their individual properties such as elastic modulus, yield stress, ductility, thermal expansion coefficient. The geometrical factors of the film such as length, width, thickness, shape are also important factors to be taken into consideration. Various combinations of thin metal film and polymer substrate are possible and new materials are being developed actively. However, the fundamental investigation of thin metal film/polymer substrate structures is still important.

In this paper, after reviewing the deformation and fracture of thin metal film on polymer substrate briefly, cracking behavior on the surface of small roundedrectangular thin gold ( $\mathrm{Au}$ ) films called "strips" deposited on plate specimens of polycarbonate (PC) was observed during cyclic and stepwisely-increased trapezoidal tensions. The effect of strip width and thickness on the cracking behavior and the crack initiation from the initial defect were discussed.

\section{Deformation and fracture of thin metal film on polymer substrate}

Deformation and fracture of thin metal film/polymer substrate structure seem to be very complex because various phenomena, such as cracking, necking, buckling, delamination and their combinations, occur. Firstly, these phenomena are briefly reviewed, and the influencing factors and their relations are clarified from the viewpoint of mechanical behavior of materials.

\subsection{Deformation, cracking and delamination of thin metal film}

Figure 1 shows the deformation of thin metal film under tension in two cases: (a) freestanding film and (b) film bonded on the surface of substrate. When the exact mechanical properties of thin film is required, mechanical tests using freestandning films are preferable $[3,4]$. When a freestanding film is subjected to tension, deformation of the film is not constrained and the localized necking easily occurs. On the other hand, if the film is bonded on the substrate, the local deformation is constrained by the substrate and uniform deformation is continued [5]. But if the substrate is very soft, deformation of the film is possibly similar to that of freestanding one. It can be understood that the deformation of the film is strongly affected by the mechanical properties of substrate.

Typical fracture phenomena which occur in thin metal film on the substrate are shown in Figure. 2. Consider a case where the tensile stress acts on the thin metal film/polymer substrate structure shown in Figure 2(a), and the stress reaches to a certain level at which the film does not stand (i.e., further deformation of the film is impossible). Cracking occurs in a perpendicular direction to the principal stress, as shown in Figure 2(b). With increasing the tension, lots of cracks are initiated in 
similar directions, and the crack sometimes propagates into the substrate and leads to the total fracture. The edge of film is also important part in film/substrate structure because a difference of the normal stress in the film and substrate brings the shearing stress, and it takes the maximum value at the edge. Then delamination occurs there when the shearing stress on the interface reaches its shearing strength, as shown in Figure 2(c). When the compressive stress acts on the film/substrate structure, it brings the interface delamination and the film is buckled, as shown in Figure 2(d). Even when the delamination does not occur due to relatively large interface strength, the film may show a wavelike buckling pattern on the surface [6].

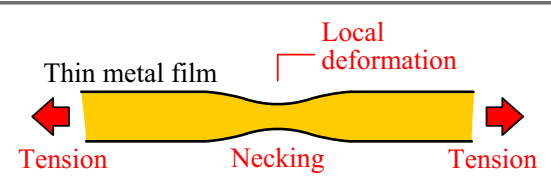

(a) Freestanding thin metal film under tension.

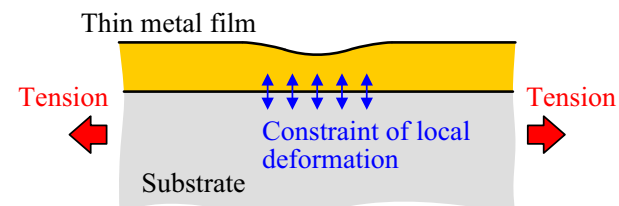

(b) Constrained deformation in thin metal film on substrate under tension.

Figure 1. Comparison of deformation of freestanding thin metal film and that bonded on substrate.

\subsection{Relationship between influential factors in the fracture of thin metal film}

Since various phenomena occur in the deformation and fracture of thin metal films on polymer substrate, their combination has to be taken into account when investigating the reliability of actual products. For example, when a crack is initiated in the thin metal film, as shown in Figure 2(b), stress concentrated at the tip promotes the local delamination of film/substrate interface. When large tension is given to the film/substrate structure, parallel cracks are initiated at almost equal distance in the film. Tensile stress in the film is released by these cracks. If the interface delamination occurs as shown in Figure 2(d), cracks are easily initiated on the tension (or outer) side of the bended thin film. The deformation, cracking, delamination are influenced each other and it makes the fracture of film/substrate structures complicated.

Figure 3 shows the relationship between influential factors in the fracture of thin metal film. Regarding the thin metal film, the thickness, shape, direction, and microstructure are typical influential factors. The microstructure influences on the mechanical properties, coefficient of thermal expansion (CTE), and strength of film. A combination of the mechanical properties and CTE determines the residual stress after deposition of the film. In order to investigate the fracture of thin metal film, each phenomenon has to be considered separately as the first step of fundamental research.

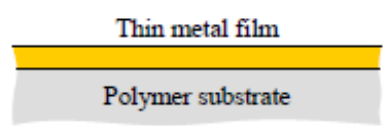

(a) Metal shin film on polymer substrate.

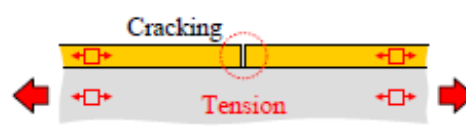

(b) Cracking in film subjected to tension.

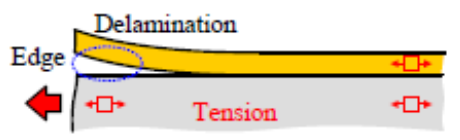

(c) Edge delamination of film subjected to tension.

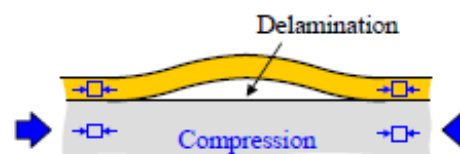

(d) Buckling of film subjected to compression.

Figure 2. Fractures occurred in thin metal film on polymer substrate.

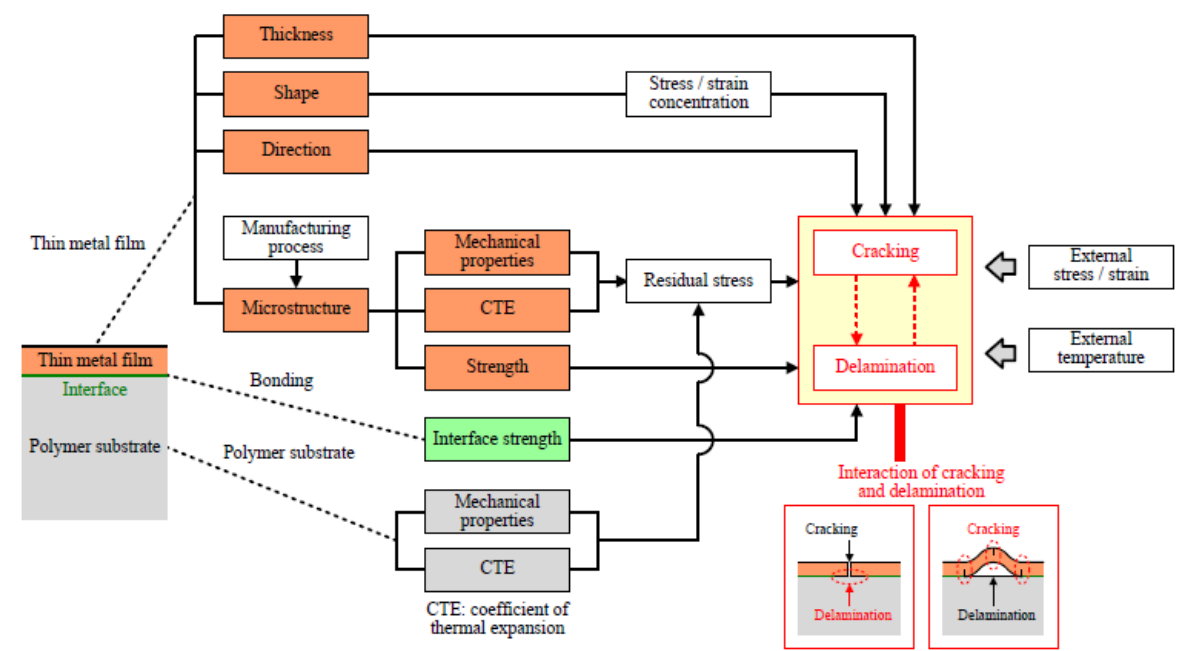

Figure 3. Relationship between influential factors in the fracture of thin metal film on polymer substrate. 


\section{Experimental procedure}

\subsection{Specimen and gold strips}

Shapes and sizes of specimen (substrate) and strip are shown in Figure 4. Transparent colorless polycarbonate (PC) plates were used for substrate and two small rounded-rectangular strips (wide strip: $2[\mathrm{~mm}] \times 1[\mathrm{~mm}]$ and narrow strip: $1.5[\mathrm{~mm}] \times 0.2[\mathrm{~mm}])$ were deposited at a parallel part of PC substrate by ion coater IB-5 (EIKO Engineering Co., Ltd.). The shape of strip was determined by putting slot grids as masking jigs. Deposition of 600 [s] with ionization current 5.5 [mA] and $300[\mathrm{~s}]$ break were repeated until the required thickness was obtained. Specimens and strips used for the tests are shown in Table 1. Since it was found that most of cracks were initiated at small defects on the strip, the relation between defects and cracks was investigated using specimens S3 and S4. The average thickness of strip, $h_{f}$, was evaluated based on the height profile of each strip measured at three lines across the strip by surface-shape measurement microscope VF-7500 (KEYENCE Corporation). The defects and cracks were observed by materials science microscope BX41M LED and confocal laser scanning microscope OLS1100 (Olympus Corporation).

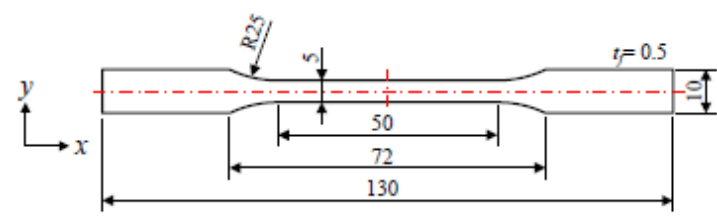

(a) Shape and size of polycarbonate specimen (substrate).

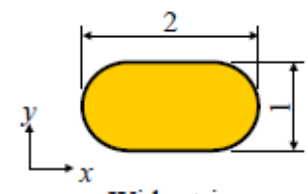

Wide strip

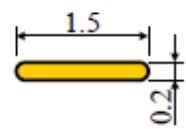

Narrow strip

(b) Shape and size of wide and narrow strips of gold.

Figure 4. Polycarbonate (PC) specimen and thin gold ( $\mathrm{Au}$ ) strip on the surface.

Table 1. Strips on each specimen.

\begin{tabular}{c|c|c|c|c}
\hline \multirow{2}{*}{ Specimen } & Strip & $\begin{array}{c}\text { Shape } \\
\text { of strip }\end{array}$ & $\begin{array}{c}\text { Strip } \\
\text { thickness }\end{array}$ & $\begin{array}{c}\text { Average } \\
\text { strip } \\
\text { thickness } \\
h_{f}[\mathrm{~nm}]\end{array}$ \\
\hline \multirow{2}{*}{ S1 } & W-L & Wide & Large & 621 \\
\cline { 2 - 5 } & N-L & Narrow & Large & 632 \\
\hline \multirow{2}{*}{ S2 } & W-S & Wide & Small & 324 \\
\cline { 2 - 5 } & N-S & Narrow & Small & 316 \\
\hline \multirow{2}{*}{ S3 } & W-L & Wide & Large & 627 \\
\cline { 2 - 5 } & N-L & Narrow & Large & 645 \\
\hline \multirow{2}{*}{ S4 } & N-S-1 & Narrow & Small & 338 \\
\cline { 2 - 5 } & N-S-2 & Narrow & Small & 314 \\
\hline
\end{tabular}

\subsection{Test conditions}

Each specimen was subjected to stepwisely-increased trapezoidal tension. The loading and unloading rate was 1 $[\mathrm{N} / \mathrm{s}]$, and the maximum load was gradually increased step by step as $P_{\max }=70,90,110$ [N]. The increment of the maximum load was decreased to $5[\mathrm{~N}]$ after these three steps. It was further decreased to 3 and $1[\mathrm{~N}]$ when very large deformation was observed. The tests were carried out using a table-top type universal tester AGS-J (Shimadzu Corporation). The observation of strip surface was carried out after each unloading.

\section{Experimental results and discussions}

\subsection{Cracking behaviour on gold strip}

Examples of cracking on wide and narrow gold strips on PC substrate are shown in Figures.5 and 6, respectively. Cracks perpendicular to the loading direction were initiated with increasing the maximum load $P_{\max }$. In the wide strip shown in Figure.5, small cracks shorter than the strip width appeared and grew to penetrate its total width. At the large maximum load, cracks were almost equally distant from each other. On the other hand, in the narrow strip shown in Figure 6, penetrated cracks appeared at the initiation and its number increased with the maximum load $P_{\max }$.

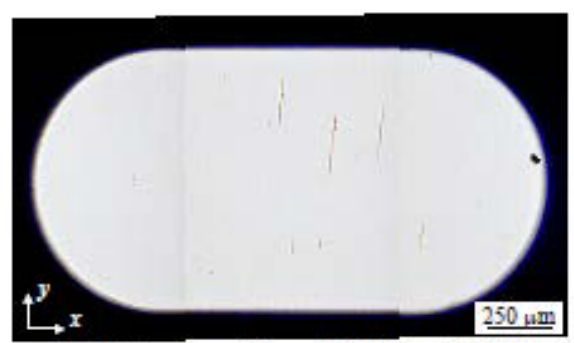

(a) After tension of $P_{\max }=145[\mathrm{~N}](s=4.0[\%])$

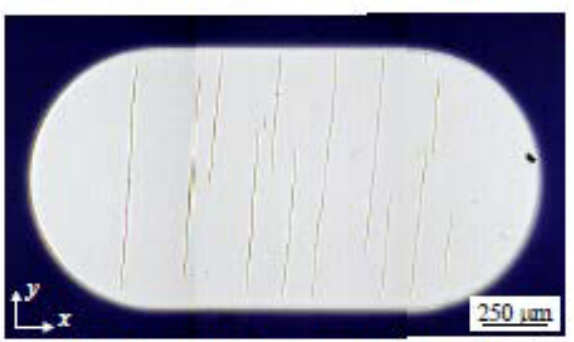

(b) After tension of $P_{\max }=150[\mathrm{~N}](\varepsilon=4.4[\%])$

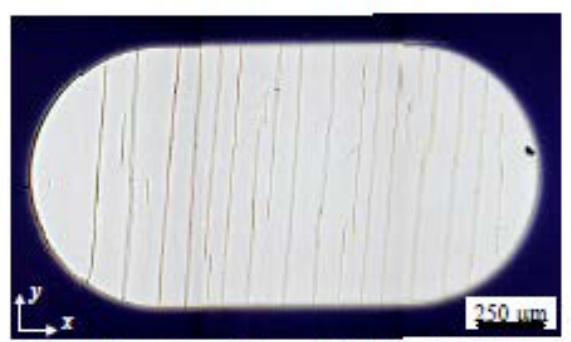

(c) After tension of $P_{\max }=160[\mathrm{~N}](\varepsilon=5.3[\%])$.

Figure 5. Cracking behavior on a thick wide strip S1-W-L. 


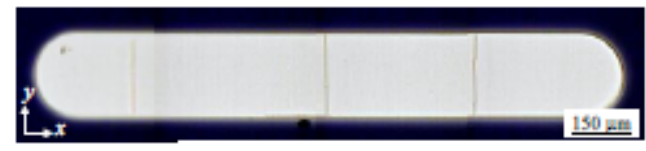

(a) After tension of $P_{\max }=150[\mathrm{~N}](\xi=4.4[\%])$.

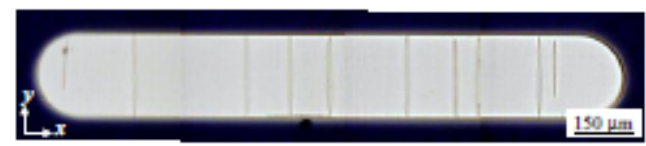

(b) After tension of $P_{\max }=155[N](\varepsilon=4.8[\%])$.

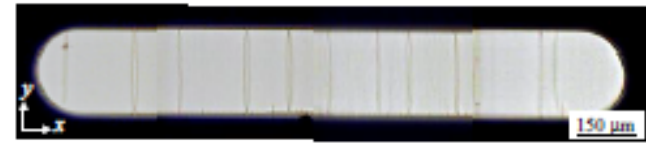

(c) After tension of $P_{\max }=160[\mathrm{~N}](\sigma=5.3[\%])$.

Figure 6. Cracking behavior on a thick narrow strip S1-N-L.

Figure 7 shows the change in crack linear density $\rho_{c}$ with the tensile strain of strip $\varepsilon . \rho_{c}\left[\mu \mathrm{m}^{-1}\right]$ was evaluated by the number of cracks $N_{c}$ divided by the longitudinal length of the $\operatorname{strip} l_{f}\left(l_{f}=2[\mathrm{~mm}]\right.$ for wide strip and $l_{f}=1.5$ [mm] for narrow strip). Use of $\rho_{c}$ minimizes the effect of the length of strip on the evaluated number of cracks. The strain $\varepsilon$ was evaluated using the finite element analysis of strip/substrate structure which was carried out to adjust the load $P$-displacement $\delta$ curve with the experimental result. It was found from Figure.7 that cracks were initiated around $\varepsilon=4[\%]$ in thick strips (S1-W-L and S1$\mathrm{N}-\mathrm{L}$ ) and $\varepsilon=6[\%]$ in thin strips (S2-W-S and S2-N-S). The effect of the strip thickness on the crack initiation strain is larger than that of strip width. On the other hand, the increasing rate of $\rho_{c}$ against $\varepsilon$ for thin strips is much larger than that for thick strips. It seems that the crack linear density curves for thin and thick strips converges on the same curve at large strain so that the strain determines the average distance between cracks.

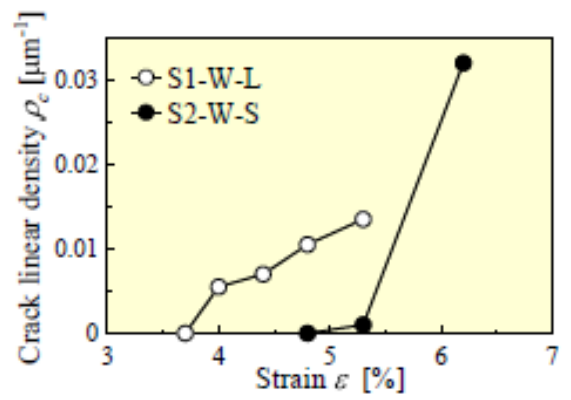

(a) Wide strips.

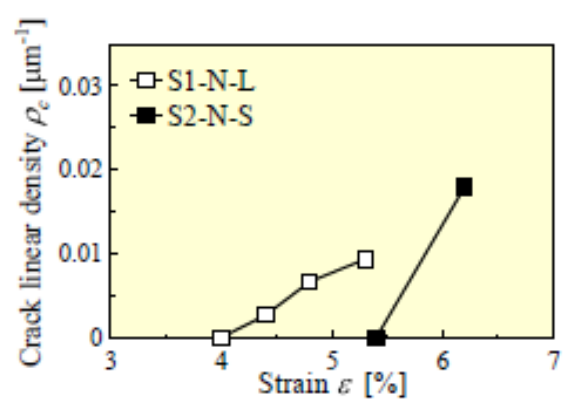

(b) Narrow strips.

Figure 7. Change in crack linear density on strips.

\subsection{Defects and crack initiation}

Magnified view of crack initiated on strip S3-N-L is shown in Figure.8. A crack was initiated from a circular defect perpendicularly to the loading direction ( $x$-axis). Most of cracks were initiated from circular defects which were thought to be formed during the deposition process. Fine vertical stripe in $y$-axis direction, which was found before and after the tension, is very small undulations on the surface of PC substrate.

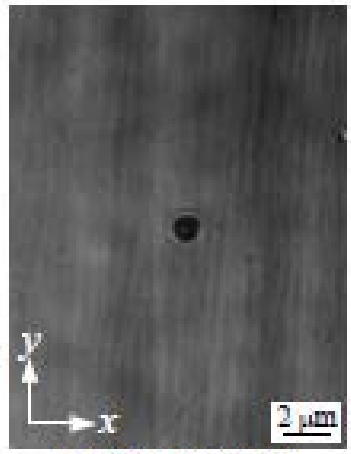

(a) Before tension

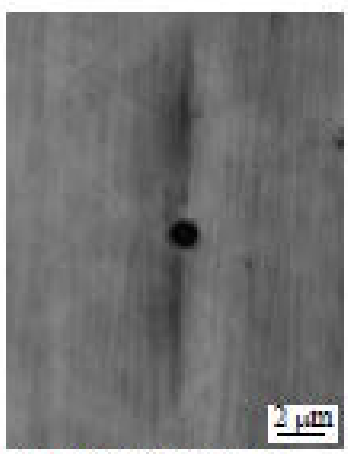

(b) After tension of $P_{\max }=151[\mathrm{~N}](\varepsilon=4.9[\%])$.
Figure 8. Crack initiated at a defect on strip S3-N-L.

The density of defects $m\left[\mu \mathrm{m}^{-2}\right]$, which is defined as the number of defects in a unit area on thin gold strip, is shown in Figure.9. An additional thick narrow strip S5N-L was used to obtain the distribution of defects. The density of defects was about $800\left[\mu \mathrm{m}^{-2}\right]$ and constant independently of the thickness of strips.

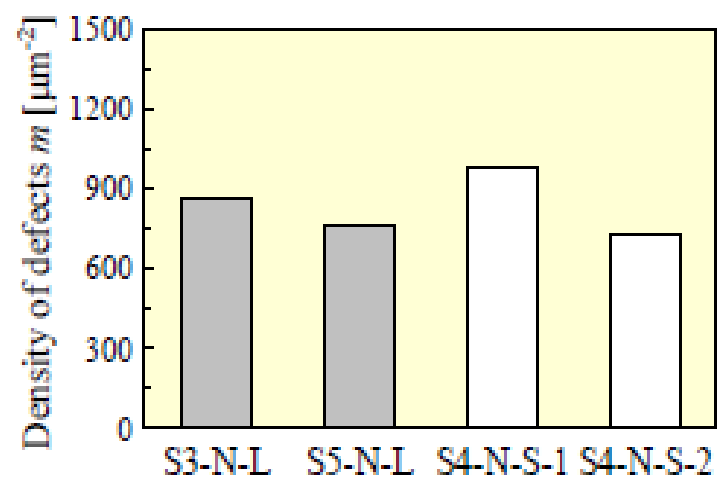

Figure 9. Density of initial defects on strips.

The distribution of equivalent diameter of defects, $d_{e q}$ $[\mu \mathrm{m}]$, is shown in Figure.10. $d_{e q}$ is defined as the diameter of circle whose area is equal to the defect. While the density of defects $m$ was almost constant in thick and thin strips, the size distribution is different. Large defects were found only on thick strips. This is one of the reasons why cracking in thick strip occurred at lower strain than the thin strips. Considering the tendency that the crack linear density for thin and thick strips converged with each other at large strain, it is concluded that cracks are easily initiated at larger defects but its number is finally controlled by the strain. 

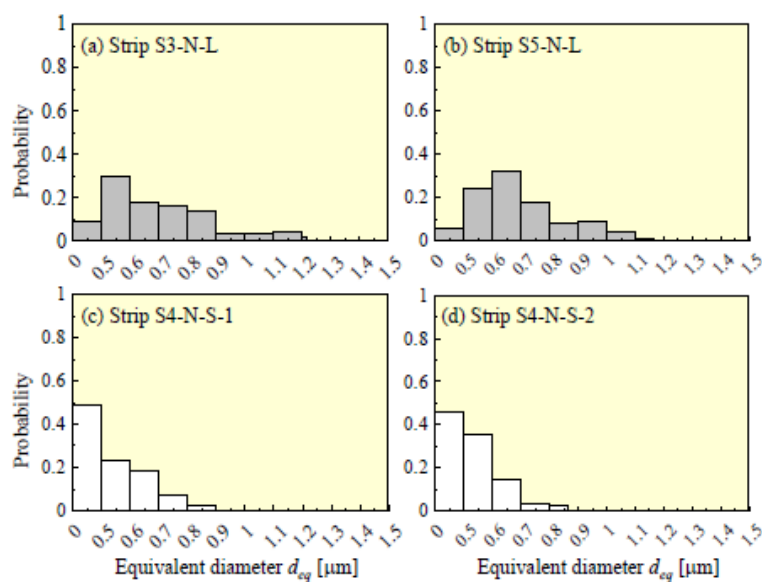

Figure 10. Distribution of equivalent diameter of initial defects on thick and thin strips.

\section{Conclusions}

Small rounded-rectangular thin gold films called "strips" were deposited on plate specimens of polycarbonate. Cracking behavior of the strips was observed during cyclic and stepwisely-increased trapezoidal tensions. Parallel cracks were observed in every strip and the initiation in thick strip was earlier (at smaller tensile strain) than in thin strip. The reason is considered to be the difference of defects' size on the strip which were formed during the deposition process.

\section{References}

1. V. J. Lumelsky, M. S. Shur, S. Wagner, IEEE Sensors Journal, 141 (2001)

2. J. A. Rogers, T. Someya, Y. Huang, Science, 327 1603 (2010)

3. S. J. Lee, J. M. Park, S. W. Han, S. M. Hyun, J. H. Kim, H. J. Lee, Exp. Mech. 50643 (2010)

4. B. Merle, M. Göken, J. Mater. Res. 29267 (2014)

5. T. Li, Z. Suo, Int. J. Solids Struct., 432351 (2006)

6. N. Bowden, S. Brittain, A. G. Evans, J. W. Hutchinson, G. M. Whitesides, Nature, 393146 (1998) 